\title{
Delitos contra el patrimonio genético nacional desde la perspectiva del COESCCI
}

\section{Crimes against the national genetic heritage from the COESCCI perspective}

\author{
Sorily Carolina Figuera Vargas \\ Doctora en Derecho \\ Academia Mexicana de Derecho Internacional Privado y Comparado - México. \\ Correo electrónico: sorilyf@yahoo.com
}

Johana Liseth Robles Arias

Abogada

Pontificia Universidad Católica del Ecuador, Quito.

Correo electrónico: johana-ra17@hotmail.com

\section{Resumen}

En Ecuador se han dado varios casos en los que empresas extranjeras han patentado material genético nacional para el desarrollo de nuevas invenciones sin contar con la debida autorización del Estado ecuatoriano. Este delito conocido como biopiratería tiene consecuencias graves a nivel ambiental, económico, social y cultural. Para comprender el alcance de este delito es necesario tener presente que mediante los recursos genéticos se puede generar bienes y servicios útiles para el ser humano, por ejemplo: el desarrollo de materias primas, biotecnología, medicinas naturales, etc. Los recursos genéticos son todo material genético de valor real o potencial, incluido el de las plantas, animales y microorganismos (Convenio sobre Diversidad Biológica, 1992). En el 
presente artículo se hace un análisis del marco legal nacional e internacional específicamente con relación a la protección de la flora y fauna del Ecuador, para determinar si esta normativa es eficiente al momento de evitar el acceso y aprovechamiento ilegal a estos recursos genéticos que tiene Ecuador. Debido a que los recursos genéticos son propiedad del Estado que los contiene, es necesario regular su uso y aprovechamiento para proteger estas especies. Finalmente, hacemos un análisis de los resultados del Primer Informe sobre Biopiratería en el Ecuador (2016) por parte del Instituto Ecuatoriano de Propiedad Intelectual para determinar el grado de afectación.

\section{Palabras clave}

Biopiratería, diversidad biológica, patrimonio genético nacional, valores de la biodiversidad, recursos genéticos.

\section{Abstract}

In Ecuador, there have been several cases in which foreign companies have patented national genetic material for the development of new inventions without the proper authorization of the Ecuadorian state. This crime, known as biopiracy has severe consequences on an environmental, economic, social, and cultural scale. To understand the scope of this crime, it is necessary to keep in mind that through the genetic resources, it is possible to generate goods and services useful for human beings, such as the development of raw materials, biotechnology, natural medicines, etc. Genetic resources are all genetic material of real or potential value, including that of plants, animals, and microorganisms (Convention on Biological Diversity, 1992). In this paper, the national and international legal framework is analyzed, specifically regarding the protection of the fauna and flora of Ecuador, to determine if this regulation is efficient at preventing the illegal access and exploitation of these genetic resources in Ecuador. Given that genetic resources are the propriety of the containing state, it is necessary to regulate their usage and exploitation to protect these species. Finally, we analyze the results from the Primer Informe Sobre Biopiratería en el Ecuador, (First report on Biopiracy in Ecuador) 2016, by the Instituto Ecuatoriano de Propiedad Intelectual (Ecuadorian Institute on Intellectual propriety) to determine the degree of impact.

\section{Key Words:}

Biopiracy, biological diversity, national genetic heritage, biodiversity values, genetic resources.

\section{Cómo citar este artículo:}

Figuera, S. C., \& Robles, J. L. (2020). Delitos contra el patrimonio genético nacional desde la perspectiva del COESCCI. Revista de la Facultad de Derecho y Ciencias Políticas, 50 (132), pp. 80-99. doi: http://dx.doi.org/10.18566/rfdcp.v50n132.a04

Recibido: 09 de julio de 2018

Aprobado: 19 de septiembre de 2019 


\section{Introducción}

Ecuador es uno de los países megadiversos del mundo, por tanto, las ventajas de este país son precisamente una gran diversidad de plantas y animales, dado que cuenta con especies endémicas, únicas en el mundo como la tortuga Galápagos, el piquero de patas azules, entre otras especies (Galapagos Conservation Trust, 2017). Esta riqueza ha sido amenazada, debido a que existen diversos casos de comercialización por parte de empresas transnacionales de productos derivados de material genético de especies de flora y fauna sin la debida autorización del Estado ecuatoriano.

Para evidenciar este delito se hace mención de un artículo del Telégrafo (periódico ecuatoriano) titulado: En 11 países se tramitan 120 patentes con recursos genéticos sustraídos de Ecuador (2016), con el propósito de comprender cómo la biopiratería causa perjuicios a los Estados que han sido afectados. En este artículo se alude a algunos ejemplos de patentes de productos generados con acceso ilegal a recursos de material genético de flora y fauna del Ecuador, que se han otorgado en el extranjero sin contar con la debida autorización del Estado Ecuatoriano.

Tal es el caso del Tomatillo endémico de Galápagos, que fue sustraído luego de estudios realizados a la planta por científicos estadounidenses, quienes se llevaron las semillas a Estados Unidos tras descubrir que genéticamente son resistentes a las plagas. Estas propiedades encontradas en el Tomatillo Endémico de Galápagos, la hace muy atractiva para investigadores y científicos. Es así que esta planta endémica ecuatoriana tiene en trámite 21 patentes en Estados Unidos (Primer Informe Sobre Biopiratería en el Ecuador, 2016).

\section{Concepto de recurso genético}

Según el abogado Lenin Núñez ,ex- director del Departamento de Biodiversidad del Ministerio del Ambiente del Ecuador, el Patrimonio Genético Nacional comprende los componentes genéticos y bioquímicos presentes en la flora y fauna de los Estados. Todas las especies forman parte de los ecosistemas, donde cumplen un rol en específico. Con todo, se debe tomar en cuenta que debe haber una protección integral para evitar su sobrepoblación. Además, no se protegen a las especies nativas de una forma especial; sino que todas las especies de plantas y animales tienen la misma importancia, por lo que se les da la misma protección desde el derecho. Por tanto, los Estados 
deben procurar una protección integral de especies: nativas, endémicas y migratorias. Para esto, se puede seguir un procedimiento de acceso con fines comerciales o investigativos, según sea el caso con estricta observancia de las normas nacionales e internacionales. (Nuñez, 2017)

Resulta importante hacer una distinción entre recurso genético y recurso biológico. El recurso biológico es la planta o animal en su estado natural. En cambio, el recurso genético es el componente del recurso biológico que tiene valor real o potencial. En consecuencia, se puede decir que el recurso biológico es la planta o animal considerado en su conjunto tal como se encuentra en la naturaleza. En contraste, los recursos genéticos pueden incluir extractos de genes, extractos biológicos, etc. Son los recursos genéticos los que constituyen la base para el desarrollo de nuevas tecnologías como medicamentos y nuevas formas de conservar alimentos (Nuñez, 2017). Si bien esta descripción de recurso genético dada por el abogado Lenin Núñez en el tema de biodiversidad es la más comprensible; sin embargo, es pertinente analizar otras definiciones de recursos genéticos que se dan en la legislación internacional, al considerar que no existe mayor discusión en el tema.

El Convenio de Diversidad Biológica (CDB), en su artículo 2 define lo que es un recurso genético: "por recurso genético se entiende el material genético de valor real o potencial” (Convenio de Diversidad Biológica, 1992, art. 2). En el artículo La importancia de los recursos genéticos vegetales y animales en el desafío de convertir a Chile en una potencia alimentaria $y$ forestal se esclarece lo que se deduce por valor real o potencial: "Que los recursos genéticos tengan un valor real o potencial significa que tienen un amplio potencial de uso, ya sea para la agricultura y la alimentación como para el sector industrial y farmacéutico; para biorremediación, defensa sanitaria, adaptación al cambio climático, entre otros.”(Agüero, 2009, p.1).

\section{Antecedentes previos a normar el acceso a los recursos genéticos}

Es significativo realizar un resumen sobre los antecedentes previos a normar el uso, acceso y explotación responsable de los recursos genéticos a partir de la obra Biodiversidad y Recursos genéticos de Jaime Estrella (2005). Según el autor, desde el derecho la lucha social para lograr una protección de las especies de flora y fauna se origina con la presión que ejercieron 
diversos grupos ambientalistas, que se fueron constituyendo y consolidando aproximadamente en la década de los cincuenta (Estrella, 2005).

La década de los ochenta es la que marca un hito en temas ambientales, en vista de la discusión extensa sobre la problemática ambiental, además, fue en este período cuando se plantearon varias propuestas que tenían como finalidad resguardar a las especies que forman parte del medio ambiente. Finalmente, la culminación de este proceso fue la Cumbre de las Naciones Unidas para el Medio Ambiente y el Desarrollo, celebrada en Rio de Janeiro de 1992, donde se discutieron posibles medidas para la conservación de los recursos naturales. En esta cumbre se crearon varios instrumentos internacionales: el más amplio en temas sobre biodiversidad es el Convenio sobre Diversidad Biológica (CDB) (Estrella, Manosalvas, Mariaca y Ribadeneira).

\section{Análisis de normativa internacional}

\section{- Convenio de Diversidad Biológica (CDB)}

El Convenio Sobre Diversidad Biológica abarca temas importantes como: "Soberanía de los estados sobre los recursos genéticos, facilitación de su acceso, derechos del país de origen de los recursos, consentimiento fundamentado previo, condiciones mutuamente acordadas, distribución justa y equitativa de los beneficios percibidos, derechos de los pueblos indígenas y comunidades locales” (Roca, 2016, p. 121). En consecuencia, la soberanía es un tema indiscutiblemente importante en cuestiones de acceso a material genético, debido a que son las naciones las que finalmente regulan estos temas. Con todo, no pueden desconocer los convenios internacionales de los que son parte. El Convenio Sobre Diversidad Biológica es un tratado internacional jurídicamente vinculante, por lo que debe usarse como parámetro para la normativa nacional con relación a temas de biodiversidad.

\section{- Decisión 391 Régimen común sobre acceso a los recursos genéticos}

La historia del Régimen Común Sobre Acceso a los Recursos Genéticos (Decision 391) que fue emitido el 2 de julio de 1996 en Caracas, Venezuela se respalda en la disposición transitoria de la Decisión 486 en su artículo 6 
con el mandato de aprobar un régimen común sobre acceso a los recursos biogenéticos y garantía a la bioseguridad de la subregión (Estrella et al.,). Fue importante que los Estados de Sur América acordaran establecer un régimen común de acceso a los recursos genéticos de especies de flora y fauna de la región para lograr ayudarse recíprocamente en la preservación y protección de especies. Al mismo tiempo, resulta notable unirse con la finalidad de cooperar en investigaciones y otros proyectos desarrollados a partir de los recursos genéticos a fin de potenciar su uso para la mejora de invenciones.

El proceso de consolidación del Régimen Común sobre Acceso a Recursos Genéticos radicó en la convocatoria a reuniones con la finalidad de que los países interesados de la Comunidad Andina (CAN: Colombia, Bolivia, Ecuador, Perú) pudieran dialogar participativamente y aportar con ideas para llegar a este acuerdo común. Esto tuvo lugar entre los años 1993 y 1994(Estrella et al.,).

El Régimen Común Sobre Acceso a Recursos Genéticos (Decisión 391) busca normar el acceso a los recursos genéticos y la repartición de beneficios. Lo que pretende es un régimen común de acceso entre los países miembros de la CAN y así lograr una mejor protección desde el derecho.

Pese a esto, en Ecuador eL régimen Común Sobre Acceso a Recursos Genéticos es una norma casi desconocida. Ello puede obedecer a varias razones, entre ellas: la poca o casi nula voluntad y decisión política en proteger de manera efectiva y evidente la biodiversidad y riqueza genética que posee el Ecuador. Otro factor determinante es, sin duda, la falta de información e importancia que las autoridades y gobiernos de turno le otorgan a la necesidad en proteger la riqueza natural del país. A lo que se suma, el poco desarrollo tecnológico del país en el área de la investigación biológica, que se relaciona directamente con las razones antes mencionadas, pues sino se conoce, no se sabe lo que se tiene.

Para esta investigación es esencial el análisis de las normas que tienen como finalidad la protección de los recursos de flora y fauna del país y que se encuentran dentro del Código Orgánico de la Economía Social de los Conocimientos, Creatividad e Innovación que fue discutido y aprobado el 9 de diciembre de 2016 y publicado en el Registro Oficial Suplemento No. 899. Tal es la importancia de este tema en términos ambientales y económicos, de ahí que uno de los principios de este cuerpo normativo es: "combatir la biopiratería para evitar que se roben nuestra riqueza” (2016). Este principio fue incorporado porque la extracción de recursos genéticos es un problema recurrente en Ecuador. 
Uno de los objetivos principales del Estado ecuatoriano es entonces combatir la biopiratería. Es por esto que el Instituto Ecuatoriano de Propiedad Intelectual (IEPI) conjuntamente con la Secretaria Nacional de Ciencia y Tecnología (SENESCYT) realizaron un informe basándose en una investigación exhaustiva donde se determinó que Estados Unidos, Alemania, Países Bajos, Australia, República de Corea, Israel, Bélgica y Francia son los principales países que han accedido ilegalmente a los recursos genéticos del Ecuador e incurrir así en el delito de biopiratería.

Para determinar el grado de afectación que sufre Ecuador por la biopiratería, se tomó como muestra a 6452 especies endémicas, lo que fue contrastado con las solicitudes de patente a nivel mundial. Una vez que se identificaron estas solicitudes de patente, se procedió a verificar la existencia de permisos de acceso a estos recursos genéticos. No se encontraron permisos de ninguna de las dieciséis especies que están presentes en las ciento veinte y ocho solicitudes de patentes que fueron identificadas.

Al considerar este informe, elaborado por la autoridad competente en materia de propiedad Intelectual IEPI, es lógico pensar que Ecuador a lo largo de su historia ha sido víctima de la biopiratería. Como medida de resarcimiento de derechos, el IEPI prepara medidas legales para presentar solicitudes de nulidad a estas patentes identificadas en el primer informe. Esto con la finalidad de reclamar el beneficio económico y científico que tiene nuestro país como dueño de estos recursos.

\section{Análisis de la normativa ecuatoriana}

Es importante hacer un analisis de la normativa interna de Ecuador con relación a la protección de los Recursos Genéticos para determinar si es efectiva para la protección de especies y si está de acuerdo con los convenios e instrumentos internacionales como el Convenio de Diversidad Biológica en estos temas

\section{- Constitución del Ecuador}

Es sustancial hacer un breve análisis del Art 400 de la Constitución ecuatoriana (Constitución del Ecuador, 2008, art. 400) que dispone lo siguiente: 
El Estado ejercerá la soberanía sobre la biodiversidad, cuya administración y gestión se realizará con responsabilidad intergeneracional. Se declara de interés público la conservación de la biodiversidad y todos sus componentes, en particular la biodiversidad agrícola y silvestre y el patrimonio genético del país. (Constitución del Ecuador, 2008)

Si la Constitución es la norma suprema dentro de los Estados y las demás leyes deben guardar conformidad con ella; entonces es lógico pensar que lo que se encuentra establecido en el Art. 400 de la Ley Máxima ecuatoriana (Constitución del Ecuador, 2008, art. 400), donde se establece que el Estado ejercerá la soberanía sobre la biodiversidad, es un tema trascendental para la debida protección de los recursos de flora y fauna en nuestro país.

Este artículo sin duda coincide con lo establecido por el Convenio sobre Diversidad Biológica (CDB) donde se deja en claro la plena soberanía de los Estados en materia de recursos genéticos.

La responsabilidad intergeneracional implica que, en el momento de acceder a los recursos genéticos con fines investigativos o comerciales, según sea el caso, es fundamental que este procedimiento se lo haga de una forma que no sea nocivo para la salud del medio ambiente. Esto con la finalidad de conservar los recursos naturales y prevenir en lo posible que las nuevas generaciones se vean afectadas por la disminución de recursos naturales. Más aun, se menciona que se tomará como una medida prioritaria la protección de especies en especial las especies que son consideradas patrimonio de la nación.

\section{- Código Orgánico de la Economía Social del Conocimiento, Creatividad e Innovación concernientes a la protección de Recursos Genéticos y el reglamento de esta ley}

A continuación, se tratan las instituciones encargadas de regular el acceso a los recursos genéticos en Ecuador con fines investigativos o comerciales. Para lograr obtener autorización para acceder a recursos genéticos del Estado ecuatoriano con fines investigativos o comerciales es necesario acatar ciertos requerimientos emitidos por la autoridad competente. Es esencial tener presente que, con el Código Orgánico de la Economía Social de los Conocimientos 
Creatividad e Innovación (COESCCI, 2016) que fue discutido y aprobado el 9 de diciembre de 2016 y publicado en el Registro Oficial Suplemento No. 899, se instituyen los requisitos de acceso a los recursos biológicos y genéticos y se modifican las competencias de las instituciones. Es así, que la Secretaria de Educación Superior, Ciencia y Tecnología (SENESCYT) pasa a ser la autoridad acreditada para generar normativa de acceso a recursos genéticos. Con esto, la SENESCYT absorbe las competencias más importantes del Ministerio del Ambiente concernientes a estos temas.

No obstante, la SENESCYT todavía no emite ninguna normativa la presente fecha, por lo que actualmente sigue vigente los procedimientos de acceso a recursos genéticos con fines investigativos que se encuentran en el Acuerdo Ministerial 034 (Norma para suscripción de contratos de acceso a recursos genéticos) que fue aprobada el 2 de marzo del 2015 y publicado en el Registro Oficial Suplemento No. 449 en Ecuador y la Decisión 391 (Régimen común sobre acceso a los recursos genéticos) que fue aprobada el 2 de julio de 1996 en Caracas, Venezuela. Con el COESCCI (2016), al Ministerio del Ambiente le compete emitir protocolos y criterios técnicos para la conservación de la biodiversidad.

Por su parte, el Reglamento del COESCCI (2016) se limita a establecer el protocolo institucional de atención en el caso de las investigaciones científicas sobre biodiversidad. Este menciona entre los artículos 25 al 27 (COESCCI, 2016, art. 25-27) las competencias institucionales, sin precisar ningún procedimiento a realizarse para los individuos o partes interesadas científica o comercialmente, a fin de que puedan realizar procesos para obtener los permisos estales requeridos para estos casos.

En consecuencia, el Reglamento del COESCCI (2016) es deficiente y no establece los procedimientos a realizarse para obtener los permisos de acceso a recursos genéticos. Sin embargo, hay que tener presente que un reglamento está subordinado a la ley y desarrolla el contenido de la misma. Por lo que si existen dudas en este reglamento entonces hay que regirse al COESCCI (2016) y a la normativa internacional.

Es fundamental analizar el procedimiento con el que actualmente se está rigiendo el acceso a recursos genéticos, para que una vez emitida la nueva normativa por parte de la SENESCYT se pueda hacer una comparación para determinar si, por ejemplo, hay un progreso en la protección de la biodiversidad en Ecuador y otros temas notables. 
Para acceder a estos recursos es necesario adherirse al procedimiento establecido en el Acuerdo Ministerial 034 de fecha 2 de marzo del 2015 publicado en el Registro Oficial Suplemento No. 449 donde se establece la normativa para suscripción de contratos de acceso a recursos geneticos. Antes de ello, estos requisitos deben presentarse ante la Dirección Nacional de Biodiversidad del Ministerio del Ambiente. En dicho acuerdo se establece que:

Las personas naturales o jurídicas debidamente acreditadas para realizar investigación científica por la Autoridad Nacional Competente, deberán presentar una solicitud ante la Dirección Nacional de Biodiversidad para la suscripción de un Contrato Marco de Acceso a los Recursos Genéticos. (Acuerdo Ministerial 034, 2015, art. 3)

El Ministerio del Ambiente emitió un formato Macro de Acceso a Recursos Genéticos, que debe ser completado por escrito. Este formulario contiene varios requisitos como : designacion de autoridad ante quien se presenta dicha solicitud, denominacion y duracion del proyecto, etc.

Hay que tomar en cuenta que las solicitudes son recibidas por las Direcciones Provinciales de la Autoridad Ambiental Nacional, quienes remitirán la información y documentación a la Dirección Nacional de Biodiversidad en un plazo no mayor a tres (3) días (Acuerdo Ministerial 034, 2015, art. 7).

La Direccion Nacional de Biodiversidad tiene el plazo de 5 días para determinar si admite o no a tramite la solicitud. Si cumple con los requisitos se procederá a una evaluación técnica (Acuerdo Ministerial 034, 2015, art.8).

En el término de 5 días la autoridad Nacional de la Biodiversidad elaborará un informe técnico sobre la viabilidad del proyecto. La Dirección Nacional de Biodiversidad en el plazo de 5 días contados, a partir de la apertura del expediente, elaborará el proyecto de Contrato Marco de Acceso a los Recursos Genéticos y remitirá a la Coordinación General Jurídica para el análisis, viabilidad y posterior suscripción del contrato de ser el caso (Acuerdo Ministerial 034, Art. 8). Finalmente, Apertura del Expediente. En el plazo de dos (2) días contados a partir de la aceptación de la solicitud, se creará el expediente con los documentos e información remitida por el solicitan (Acuerdo Ministerial 034, 2015, art.9).

Estos requisitos corresponden a la normativa interna de nuestro país. Para determinar si esta normativa esta deacuerdo o no con los parámetros 
establecidos en la "Decision 391 de acceso a recursos geneticos", con los que deben regirse los paises miembros, es necesario realizar un cuadro ejemplificativo en el cual se estableceran los principios comunes de acceso (véase posteriormente Gráfico 1).

Finalmente, puede manifestarse que la normativa de regulación de suscripción de contratos marcos para el acceso a recursos genéticos constituye un conglomerado procedimental en la que se establecen los formalismos que se deseen observar en el proceso de solicitud y celebración de un contrato para el acceso a los recursos genéticos. Si bien la normativa guarda una relación compatible con lineamientos del Regimen Comun Sobre Acceso a Recursos Geneticos de la Comunidad Andina, en su contenido se limita justamente a la mencionado, sin establecer una mayor seguridad que resguarde la propiedad genética del estado ecuatoriano. Tampoco se establece, por ejemplo, que dentro de las investigaciones científicas llevadas a cabo por personas naturales o jurídicas extranjeras se deba contar con la participación de un mínimo de personal científico ecuatoriano.

Esto con el fin de que, por un lado, se logre un mejor control del cumplimiento del contrato marco.Por otro , en aras de dotar al personal científico ecuatoriano de experiencias que permitan enriquecer el acevro académico y científico tanto personal como institucional del Ecuador.

Un aspecto positivo a destacar del acuerdo ministerial para suscripción de contratos de acceso a recursos genéticos es el convenio de transferencia de material genético, ámbito donde el Ecuador posee varias garantías, así como mantiene una propiedad permanente sobre los recursos genéticos que sean objeto de investigación científica y que pretendan ser apropiados por otras personas ajenas a los intereses del país.

Se presenta a continuación el flujograma del proceso de aceleración del contrato marco: 
Grafico 1. Principios Comunes de Acceso a los Recursos Genéticos

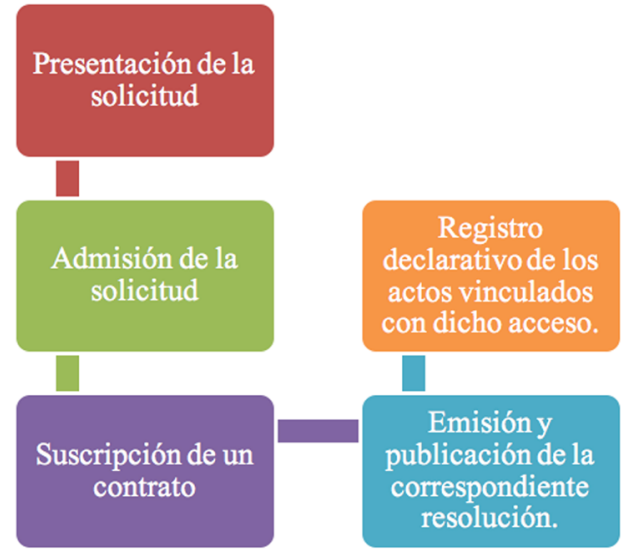

Fuente : Parametros establecidos en la Decisión 391 en el Titulo V Capitulo 1 titulado: "Del Contrato de Acceso". Elabordo por: Johana Robles.

Al hacer un análisis comparativo de la normativa interna y la Decisión 391, Ecuador sí se adhiere a la normativa de acceso a recursos genéticos con fines investigativos. Por tanto, la normativa que tiene que presentar la SENESYT para el acceso a recursos genéticos, de acuerdo al Dr. Lenin Nuñez de la Dirección Nacional de Biodiversidad, no sufrirá cambios extremos. Entonces, los requisitos esenciales seguirán manteniéndose con la nueva normativa de acceso a recursos genéticos. Sin embargo, hay que tener presente que en el Art. 25 del Reglamento al COESCCI (COESCCI, 2016, art. 25) se establece un procedimiento más sencillo, debido a que las solicitudes se receptarán de forma virtual (Decreto Ejecutivo 1435).

Ahora es necesario hacer un análisis de la normativa vigente en Ecuador con respecto al acceso a recursos genéticos con fines comerciales, hasta que la SENESCYT emita una nueva normativa y entonces éstos queden derogados. Esto tendrá la finalidad de determinar si actualmente existe una participación justa y equitativa de los beneficios derivados del acceso.

Prioritariamente es necesario tener presente las partes que intervienen en el contrato, que tiene como finalidad el acceso a los recursos genéticos con fines comerciales. El Estado es representado por la autoridad Nacional competente y el solicitante de acceso son las partes que intervienen en el contrato de acceso (Régimen Común Sobre Acceso a los Recursos Genéticos, Decisión 391, 1996, 
art. 32). Los contratos de acceso, además, deben incluir condiciones como: la participación justa y equitativa de los beneficios obtenidos a partir del recurso, cuando este se encuentre en territorio de los pueblos y nacionalidades. Además, una parte debe destinarse a la restauración y protección de la biodiversidad dentro del territorio (Decisión 391, Art. 32, 1996). Esto con la finalidad de una distribución justa de los beneficios obtenidos a partir del acceso a los recursos genéticos.

Esta repartición de beneficios está orientada a reconocer económicamente, mediante la comercialización, los frutos de la investigación científica; pues esta lógicamente demanda un esfuerzo intelectual y material para la investigación y obtención de resultados. Por otro lado, se reconoce también que los poseedores de esos recursos han de ser retribuidos de igual manera, por ser justamente los titulares de los derechos que sobre ese patrimonio reconoce la ley y el Estado.

Según Lenin Núñez (2017) de la Dirección Nacional de la Biodiversidad, actualmente no existe una participación justa y equitativa de los beneficios obtenidos de la biodiversidad, debido a que el Estado participa en un porcentaje mayoritario de los réditos que generen los inventos, a partir de los principios activos para la consecución de inventos biotecnológicos como algunos fármacos.

El Estado tiene un aporte mayoritario a pesar de que no hace nada, solo permite el acceso al recurso genético. La investigación y los gastos que conlleva corren por cuenta del investigador. Para que un medicamento sea aprobado es necesario realizar varios procedimientos en laboratorios y otras pruebas que son muy costosas. Todo esto lo debe hacer el investigador y el Estado es el que tendrá un beneficio mayoritario si estos medicamentos salen al mercado.

Esta prerrogativa favorable al Estado ecuatoriano podría estar fundada en varios argumentos: Por un lado, al ser el Estado la persona ficticia que encarna la soberanía del pueblo y la sociedad justamente vela por el interés común, aquello que más favorezca al conglomerado que representa. En este sentido se justificaría esa participación hegemónica del Estado en los beneficios que se obtienen a partir de las investigaciones científicas con fines comerciales sobre los recursos genéticos y de biodiversidad que posee el país.

Por otro lado, podría entenderse también que esta supremacía estatal frente a los beneficios de la investigación científica, cuyo producto es la elaboración de diversos tipos de bienes como medicamentos, puede ser un elemento que 
desanima la investigación científica; puesto que la inversión que se realiza en una acción de este ámbito es, por lo general, costosa y de larga duración. Por supuesto, esto es una inversión onerosa para el investigador y/o la entidad que auspicia la investigación.

En este sentido, la ley podría establecer un mecanismo de repartición de beneficios que sea atractiva para los investigadores, en cuanto a los beneficios económicos ulteriores que podamos tener como producto de su investigación científica y aplicaciones de la misma. Esto no quiere decir que el Estado seda su soberanía biológica; sino que más bien sea un facilitador participativo, que incentive la investigación científica de los recursos biológicos y genéticos del país, al otorgar beneficios justos y atractivos tanto para el investigador como para el estado; pues de esta manera se perdía un impulso a la investigación científica de aprovechamiento responsable de los recursos genéticos que posee el Ecuador para beneficio de su población, del Estado, y de la humanidad.

Esta reflexión del Dr. Lenin Núñez de la Dirección Nacional de la Biodiversidad es acorde al Art. 408 de la Constitución Ecuatoriana del 20 de octubre de 2008 (Constitución ecuatoriana, 2008, art. 408), publicada en el Registro Oficial No. 449, al establecer que: "El Estado participará en los beneficios del aprovechamiento de estos recursos, en un monto que no será inferior a los de la empresa que los explota” (Constitución del Ecuador, 2008).

La norma constitucional, como puede evidenciarse, insta a que la participación en los beneficios producto de las investigaciones científicas en la biodiversidad y recursos genéticos, puede ser del 50\% para cada uno de las partes. De hecho, esta disposición podría ser considerada justa; sin embargo, como ya se mencionó , la investigación científica es ponderosa y a largo plazo, por lo que podría determinarse en la ley el otorgamiento de ciertas facilidades por parte del Estado en beneficio de los investigadores, a fin de incentivar este tipo de labor en el país. Esto conforme a la necesidad de transmisión de tecnología hacia el Ecuador, así como uno de los aspectos puede aportar a la economía de Estado.

En el COESCCI (2016) se establece que en la normativa que debe emitir la SENESYT deberá prever una participación justa y equitativa de los beneficios que se obtengan de la biodiversidad. Con todo, como se indicó todavía no existe esta normativa y, una vez que esta publique, se podrá hacer un análisis sobre si existe un avance o un retroceso en estos temas. 
Se concuerda con el Dr. Lenin Núñez con respecto a que la normativa va a permanecer igual y simplemente se cambia las competencias. Sin embargo, el Código de la Economía Social de los Conocimientos publicado en el Registro Oficial en el año 2016 propone nuevos temas y trata de avanzar en la regulación de acceso de especies animales y vegetales.

En el Art.73 del COESCCI se establece que: "los beneficios percibidos serán destinados según la política pública determinada por la Secretaria de Educación Superior, Ciencia Tecnología e Innovación, la cual en todos los casos deberá prever un porcentaje mayoritario para, ciencia, tecnología e innovación” (COESCCI, 2016, art. 173) . Además, se indica que el Estado percibirá al menos la misma cantidad de beneficios monetarios y no monetarios que otros actores.

Al analizar la norma y por la entrevista que tuvimos con el ex director del Departamento de Biodiversidad del Ministerio del Ambiente del Ecuador Lenin Núñez el 16 de mayo del 2017, se puede decir que las políticas de la SENESCYT no cambiarán mucho en estos temas y el Estado seguirá teniendo una participación mayoritaria con relación a los beneficios de la biodiversidad. Esto es totalmente injusto, porque no se incentiva la investigación; puesto que esta exagerada prerrogativa a favor del Estado tendrá un efecto negativo en el incentivo de investigaciones científicas sobre los recursos genéticos y biodiversos del Ecuador. Debido a que el retorno de la inversión no solo es a largo plazo; sino que demanda una inversión cuantiosa y permanente, mientras se logra y se espera sus resultados, pues el proceso científico demanda la comprobación de los resultados.

Otro aspecto a considerar es que las solicitudes de acceso a recursos genéticos con fines comerciales se recibirán de forma virtual en la ventanilla única, al igual que las solicitudes de acceso a recursos genéticos con fines investigativos. Esto hará que el procedimiento de acceso sea más rápido y sencillo, una vez que se cree esta plataforma virtual (Decreto ejecutivo, 2017, Art. 25). Mientras se incorpora este sistema virtual, los trámites continuarán sustanciándose a través de la presentación física de las propuestas investigativas en las entidades correspondientes, como son en este caso el ministerio del ambiente y la SENESCYT.

Las disposiciones establecidas en el Reglamento al Código Orgánico de la Economía Social de los Conocimientos, Creatividad e Innovación son novedosas, debido a que se emplean las herramientas tecnológicas para agilizar los procedimientos de acceso a los recursos genéticos. Esto trata de prever 
y evitar demoras en el procedimiento para incentivar nuevas invenciones, al considerar que la investigación para el desarrollo de nuevas tecnologías toma mucho tiempo antes de ser aprobadas y salir al mercado.

\section{Análisis de los resultados del primer informe de Biopiratería en el Ecuador}

Es necesario analizar los resultados obtenidos por el Instituto Ecuatoriano de Propiedad Intelectual para determinar el grado de afectación que ha tenido el Estado ecuatoriano a causa de la biopiratería.

Con base en los resultados obtenidos por el Instituto Ecuatoriano de Propiedad Intelectual se determinó que los países que lideran las cifras de acceso no autorizado al patrimonio genético del Ecuador son los siguientes:

Gráfico 2. Acceso ilegal al patrimonio genético del Ecuador

\begin{tabular}{|l|l|}
\hline $\begin{array}{l}\text { Países que lideran las cifras de acce- } \\
\text { so no autorizado }\end{array}$ & $\begin{array}{l}\text { Número de patentes concedidas sin la } \\
\text { autorización del Estado ecuatoriano }\end{array}$ \\
\hline Estados Unidos & 35 \\
\hline Alemania & 33 \\
\hline Países Bajos & 17 \\
\hline Australia & 15 \\
\hline
\end{tabular}

Elaborado por: Johana Robles \& Sorily Figuera.

Estas cifras alarmantes muestran que efectivamente países con tecnologías más avanzadas se están aprovechando de la riqueza del país. Por esto es importante que el Estado afectado por la biopiratería solicite las nulidades de estas patentes identificadas para resarcir los daños devastadores que causa la biopiratería a nivel económico, social y ambiental.

Según el informe mencionado anteriormente, se pudo identificar las especies endémicas que han sido más afectadas por la biopiratería, tal como se ilustra a continuación: 
Gráfico 3: Especies afectadas por el delito de biopiratería

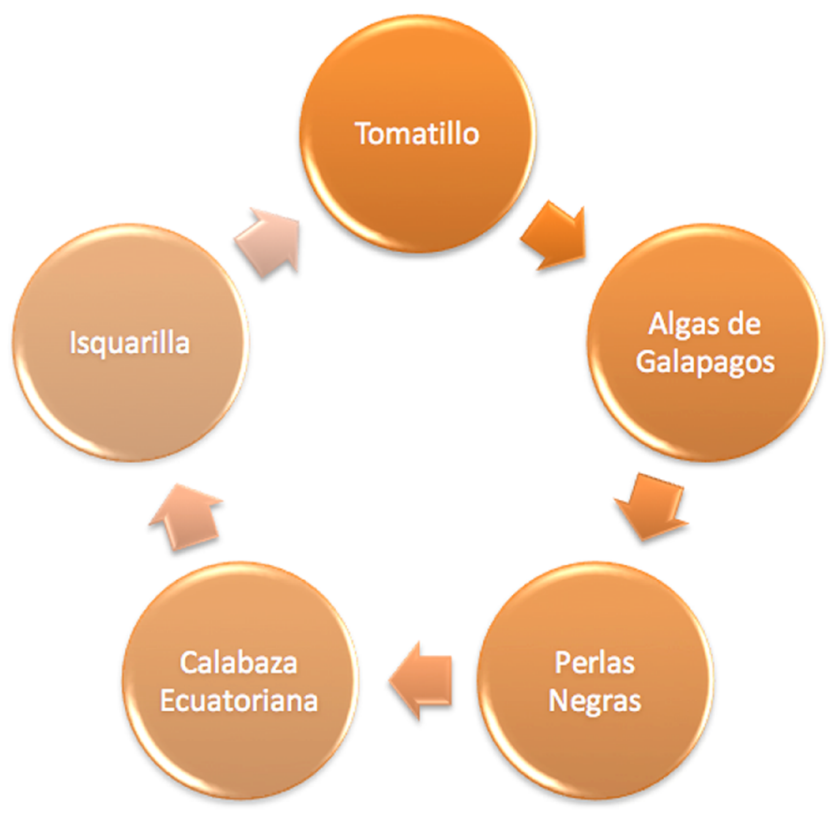

Elaborado Por: Johana Robles \& Sorily Figuera

Estas especies endémicas identificadas en el primer informe de biopiratería cumplen un rol ecológico clave, por ejemplo, el algodón de Darwin, especie endémica de las islas Galápagos, es utilizada por las aves para la construcción de nidos. En definitiva, estas especies endémicas identificadas cumplen un rol en la naturaleza y además benefician al ser humano.

\section{Conclusiones}

La biopiratería ha afectado gravemente a la soberanía del país, debido a que empresas transnacionales han patentado el material genético ecuatoriano sin contar con su autorización. En consecuencia, no habido un reparto equitativo de los beneficios derivados de las invenciones. Al considerar los resultados obtenidos en el primer informe de biopiratería se puede determinar las especies más afectadas por este delito con la finalidad de que las autoridades competentes en temas de protección ambiental puedan presentar solicitudes de nulidad de las patentes que han sido obtenidas de forma ilegal. En este 
sentido este artículo se propuso analizar las razones por las que la biopiratería causa perjuicios a los Estados, particularmente al Estado ecuatoriano; además, concientizar a los Estados para que lideren iniciativas orientadas a proteger las especies de flora y fauna y, de esta manera, reducir la pérdida de la biodiversidad.

\section{Referencias}

Secretaria del Convenio sobre la diversidad. (2011). Decenio de las Naciones Unidas sobre la Biodiversidad. Recuperado de Convenio sobre la Diversida dBIologica: https://www.cbd.int/undb/media/factsheets/undb-factsheets-es-web.pdf

Agüero, T. (2009). La importancia de los recursos geneticos vegetales y animales en el desafio de convertir a chile en una potencia alimentaria y forestal. Recuperado de: Oficina de estudios y politicas agrarias: http://www.odepa.cl/odepaweb/ publicaciones/doc/2142.pdf

Ambiente, M. d. (s.f.). UTPL. Formato para el informe anual Acceso Recursos Genéticos. Recuperado de: http://investigacion.utpl.edu.ec/es/node/166

Asamblea Constituyente. (2008). Constitución del Ecuador. Quito: Registro Oficial.

Asamblea Nacional del Ecuador. (2016). Código Orgánico de la economía social de los conocimientos, cretividad e innovación. Quito: Registro Oficial No. 899.

Bravo, E. (2014). La biodiversidad en el Ecuador. Quito: Abya-Yala.

Comunidad Andina. (2 de julio de 1996). Régimen Común Sobre Acceso a los Recursos Genéticos, Decisión 391. Lima, Perú. Recuperado de: http://www.sice.oas.org/ trade/JUNAC/decisiones/DEC391S.asp

Decreto Ejecutivo. (7 de junio de 2017). Reglamento código orgánico economía social de los conocimientos. Quito, Ecuador: Registro Oficial Suplemento 9.

Estrella, J., Manosalvas, R., Mariaca, J. y Ribadeneira, M. (2005). Biodiversidad y Recursos Geneticos: Una guia para su uso y acceso en el Ecuador. Quito: Abya-Yala.

Galapagos Conservation Trust. (noviembre de 2017). Galapagos Conservation Trust. Recuperado de:: https://galapagosconservation.org.uk/

Instituto Ecuatoriano de la Propiedad Intelectual. (15 de noviembre de 2017). Instituto Ecuatoriano de la Propiedad Intelectual. Recuperado de:: https://www. propiedadintelectual.gob.ec/

Instituto Ecuatoriano de la Propiedad Intelectual (23 de junio de 2016). Instituto Ecuatoriano de la propiedad Intelectual. Recuperdado de : https://www. propiedadintelectual.gob.ec/wp-content/uploads/2013/09/Primer-Informe-29de-junio-12-43.pdf

Ministerio del Ambiente. Norma que regula el procedimiento para la suscripción de contratos marco de acceso a recursos genéticos. Acuerdo Ministerial 034. (2 de marzo de 2015). Quito: .

Naciones Unidas. (1992). Convenio de Diversidad Biológica. (Recuperado de: http:// www.humboldt.org.co/images/documentos/pdf/Normativo/1994-ley165-1994. pdf 
Nuñez, L. (16 de mayo de 2017). Delitos contra el patrimonio biogenético del Ecuador. (J. Robles, Entrevistador).

Organización de las Naciones Unidas para la Alimentación y la Agricultura. (2001). Tratado Internacional sobre los Recursos Fitogenéticos para la Alimentación y la Agricultura. Naciones Unidas.

Organización de las Naciones Unidas para la Alimentación y la Agricultura. (noviembre de 2017). FAO Recuperado de: http://www.fao.org/home/es/

Roca, S. T. (2016). Biodiversidad y Propiedad Intelectual en Disputa. Lima: CEPIC.

Ruiz, M. (2016). Recursos Naturales y Ambiente. En Una lectura crítica de la Decisión 391. Recuperado de: http://www.planttreaty.org/sites/default/files/rna_136_147. pdf

Secretaría de Educación Superior , Ciencia y tecnología, e Inovación. (2016). Primer Informe sobre biopirateria en el Ecuador. Quito: Senescyt.

\section{CODIGOS , LEYES Y CUERPOS NORMATIVOS}

Asamblea Constituyente. (2008). Constitución del Ecuador. Quito: Registro Oficial.

Asamblea Nacional del Ecuador. (2016). Código Orgánico de la economía social de los conocimientos, cretividad e innovación. Quito: Registro Oficial No. 899.

Comunidad Andina. (2 de julio de 1996). Régimen Común Sobre Acceso a los Recursos Genéticos, Decisión 391. Lima, Perú Recuperado de: http://www.sice.oas.org/trade/ JUNAC/decisiones/DEC391S.asp

Decreto Ejecutivo. (7 de junio de 2017). Reglamento código orgánico economía social de los conocimientos. Quito, Ecuador: Registro Oficial Suplemento 9.

Ministerio del Ambiente. Norma que regula el procedimiento para la suscripción de contratos marco de acceso a recursos genéticos. Acuerdo Ministerial 034. (2 de marzo de 2015). Quito. 